\title{
Challenges in the Histopathologic Diagnosis of Brain Tumors: An Institutional Experience in a Series of Cases
}

\author{
Jayaprakash K. Shetty ${ }^{1}$ Kishan H.L. Prasad ${ }^{1}$ Shruthi S. ${ }^{1}$ Ananthan Raghothaman ${ }^{2}$ \\ ${ }^{1}$ Department of Pathology, K S Hegde Medical Academy, Nitte \\ (Deemed to be University), Mangalore, Karnataka, India \\ Address for correspondence Shruthi, MBBS, MD, Department of \\ Pathology, K S Hegde Medical Academy, Nitte (Deemed to be \\ 2 Department of Neurosurgery, K S Hegde Medical Academy, Nitte \\ (Deemed to be University), Mangalore, Karnataka, India \\ University), Deralakatte, Mangalore, DK District, Karnataka, 575018, \\ India (e-mail: shruthibelthangadi@gmail.com). \\ J Health Allied Sci ${ }^{\mathrm{NU}}$ 2022;12:412-416.
}

\begin{abstract}
Background Central nervous system (CNS) tumors are relatively rare. However, brain tumors are one of the leading causes of cancer-related morbidity and mortality. Accurate histopathologic diagnosis and molecular diagnostics are critical for managing these patients. Histopathology plays a vital role in diagnosis, but clinical and radiological information is also crucial while evaluating brain tumors.

Materials and Methods A cross-sectional observational study was performed for a period of 1 year in the pathology department of a tertiary hospital. All the brain biopsies sent for histopathological analysis were analyzed, and among which five brain biopsy tissue posing the diagnostic dilemma in conventional histopathology were included in the study. Immunohistochemistry was performed wherever necessary. Results During the study period, we encountered 32 cases of brain biopsy. Five cases posing diagnostic challenges in histopathological diagnosis were included in the study.

\section{Keywords}

- brain tumors

- angiosarcoma

- pilocytic astrocytoma

- embryonal tumor

- glioblastoma Expert opinions of neuropathologists were taken in all of these cases. We reviewed the cases for the diagnostic difficulties. A review of all these cases has impacted further treatment. Conclusion In this case series, we present five CNS tumors with a diagnostic dilemma in histopathological diagnosis where clinicoradiological correlation, immunohistochemistry, and expert opinion helped in the definitive diagnosis revealing the importance of a multidisciplinary approach for the better management of patients.
\end{abstract}

\section{Key Message}

Histopathologic assessment of central nervous system tumors remains the primary means of establishing a definitive diagnosis and predicting patient outcomes. Radiology-based provisional diagnosis should be considered during reporting, and pathologists should not hesitate to take expert opinion for the correct diagnosis and better treatment approach in these patients.

received

November 5, 2021

accepted

December 18, 2021

published online

February 9, 2022
DOI https://doi.org/

$10.1055 / \mathrm{s}-0042-1742372$.

ISSN 2582-4287.

\section{Introduction}

Central nervous system (CNS) tumors are rare, and these tumors are the second most common type of malignancy in children. ${ }^{1}$ Accurate histopathological evaluation is essential in deciding the treatment for CNS tumors. Molecular diagnostics are helpful in therapeutic decision-making and assessing the prognosis. However, these tests are very expensive and are not available in developing countries. ${ }^{2}$

\footnotetext{
(c) 2022. Nitte (Deemed to be University). All rights reserved.

This is an open access article published by Thieme under the terms of the Creative Commons Attribution-NonDerivative-NonCommercial-License, permitting copying and reproduction so long as the original work is given appropriate credit. Contents may not be used for commercial purposes, or adapted, remixed, transformed or built upon. (https://creativecommons.org/ licenses/by-nc-nd/4.0/)

Thieme Medical and Scientific Publishers Pvt. Ltd., A-12, 2nd Floor, Sector 2, Noida-201301 UP, India
} 
Evaluation of brain tumors requires detailed clinical information, radiological correlation, histological hallmarks, and immunohistochemistry. The tumor location and age of the patient are considered in the histological differential diagnosis. ${ }^{3}$ A benign neoplasm in the brain can be lethal due to its location, space-occupying effects, and the risk of malignant transformation over a while. ${ }^{4}$

\section{Materials and Methods}

A cross-sectional observational study was performed for a period of 1 year in the pathology department of a tertiary hospital. All the brain biopsies sent for histopathological analysis were analyzed, and among which five brain biopsy tissue posing the diagnostic dilemma in conventional histopathology were included in the study. Clinical, radiological, and pathological features were analyzed during the evaluation of the tumor. Immunohistochemistry was performed wherever indicated.

\section{Results}

During the study period, we encountered 32 cases of brain biopsy. Five cases posing diagnostic challenges in histopathological diagnosis were included in the study. Expert opinions of neuropathologists were taken in all of these cases. We reviewed the cases for the diagnostic difficulties. A review of all these cases has impacted further treatment.

\section{Case 1}

A 25-year-old male came with a right-sided weakness for 6 months. Magnetic resonance imaging (MRI) showed a dural-based extra-axial mass with overlying calvarial destruction. Biopsy from the temporoparietal region lesion showed highly pleomorphic cells in sheets lining the proliferating blood vessels. Many atypical mitotic figures, necrosis, and bleeding were present with the histopathologic diagnosis of anaplastic meningioma (World Health Organization [WHO] Grade 3 ). The tissue was sent to a referral center where immunohistochemistry showed CD31, Friend leukemia integration 1 (FLI) positivity in tumor cells (-Fig. 1); hence the pathological diagnosis of epithelioid angiosarcoma was made. The patient was treated with resection of the tumor, followed by radiotherapy. The patient was on regular follow-up and presented with a recurrence after 6 months, after which the patient was on palliative care. Later the patient succumbed.

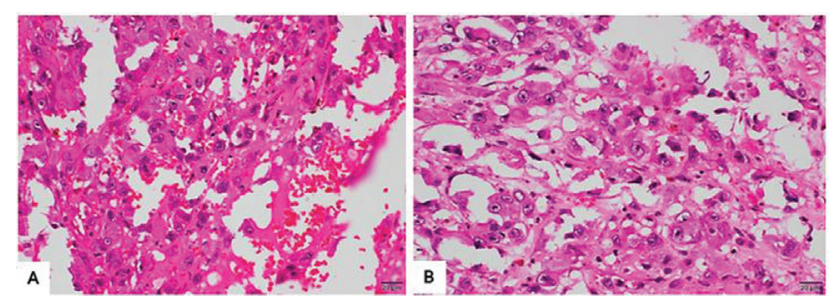

Fig. 1 Epithelioid angiosarcoma (Case 1): (A, B) Pleomorphic epithelioid tumor cells often lining the blood vessels (hematoxylin and eosin, $\times 400)$.

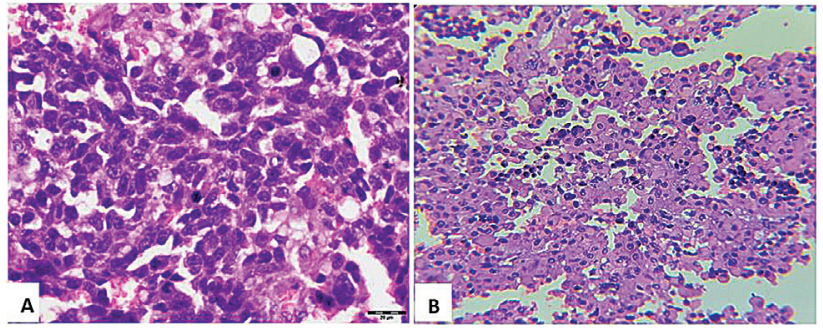

Fig. 2 Embryonal tumor with rhabdoid morphology (Case 2): (A) Highly cellular tumor with undifferentiated cells and increased mitotic activity (hematoxylin and eosin, $\times 400$ ). (B) Tumor cells with rhabdoid morphology (hematoxylin and eosin, $\times 200$ ).

\section{Case 2}

Two-year-old male child presented with one to two episodes of seizure in 2 days. MRI showed features of high-grade glioma. Biopsy showed undifferentiated tumor cells. Many spindly cells with rhabdoid morphology were present. Large areas of necrosis, brisk mitotic activity, focal fibrillary background, occasional Homer Wright, and true rosettes were noted. Diagnosis of glioblastoma/embryonal tumor was made and was sent for neuropathologist opinion. Immunohistochemistry showed LIN28, epithelial membrane antigen (EMA), and vimentin positivity. INI1 (SMARCB1) was retained. BRG1 (SMARCA4) was advised because of rhabdoid cells with retained INI1, which was not performed (-Fig. 2). Based on immunohistochemistry, diagnosis of the embryonal tumor with the rhabdoid feature (WHO grade 4) was made. The patient underwent left frontal craniotomy with excision. Postoperatively the patient was symptomatically better and discharged with antiepileptics. Advised for regular follow-up, the patient is doing well.

\section{Case 3}

A 35-year-old male presented with headache and heaviness in the head in the last 8 months. MRI showed hydrocephalus with posterior fossa space-occupying lesion. Biopsy showed predominantly hyalinized blood vessels with sparse bipolar benign cells in a fibrillary background. Extensive calcification with psammoma bodies, and large hemorrhagic foci were also present (-Fig. 3). Diagnosis of pilocytic astrocytoma (WHO grade 1) was made based on additional clinical information and clinical differential diagnosis. The tumor was resected entirely, and the patient was discharged. The patient is on follow-up, and there has been no recurrence so far.

\section{Case 4}

A 68-year-old male presented with right-sided weakness and headache in the past month. MRI was suggestive of high-grade glioma in the left frontotemporal cortex. The patient also had a nonhealing ulcer over the left great toe in the past 2 years. Biopsies from both temporal cortex and leg ulcer were sent for histopathology. The temporal cortex lesion showed tumor cells in sheets, nests, and glomeruloid vascular proliferation. The tumor cells were pleomorphic, having an atypical nucleus with 


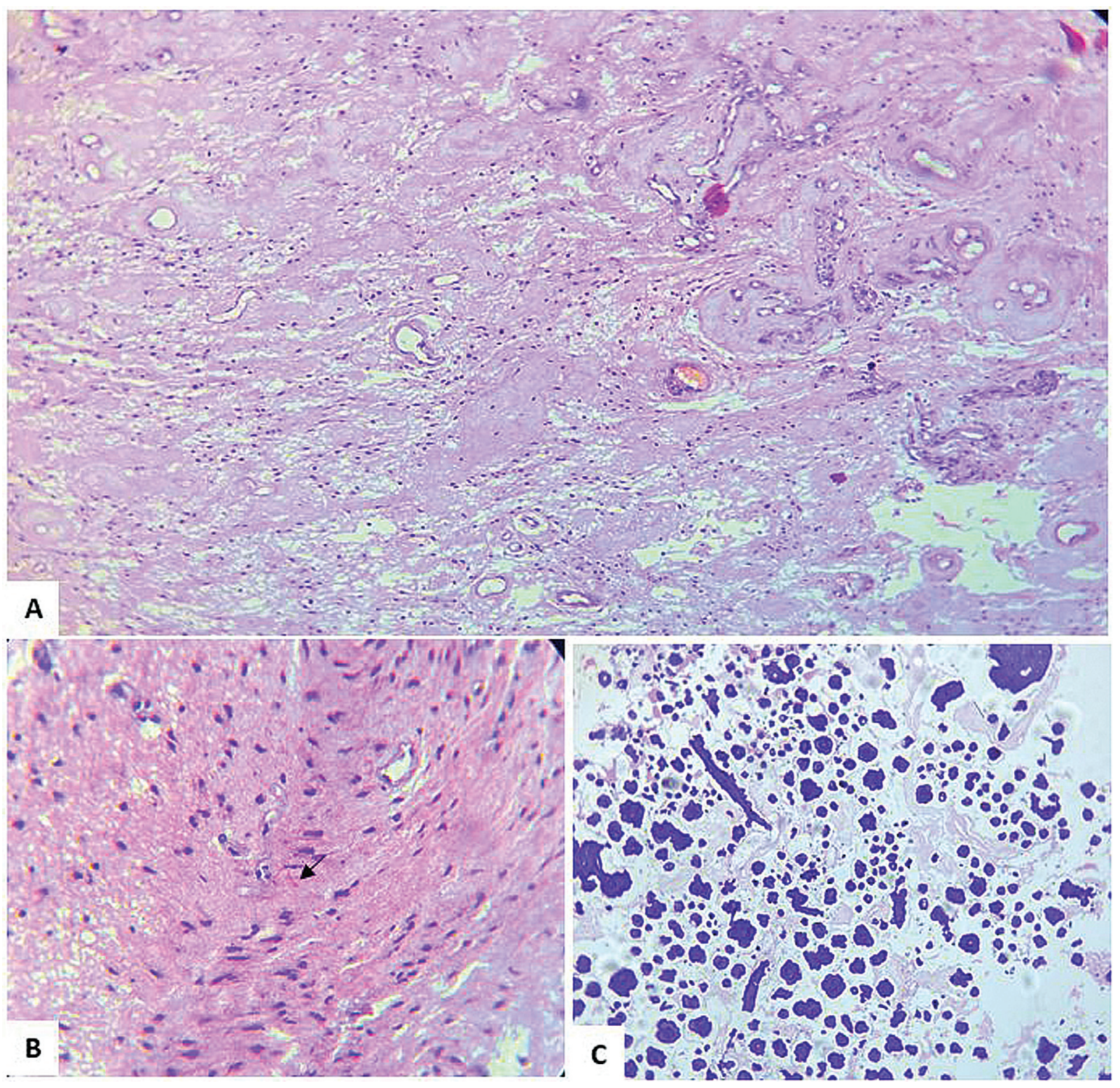

Fig. 3 Pilocytic astrocytoma (Case3): (A) Prominent hyalinized blood vessels (hematoxylin and eosin, $\times 100$ ). (B) Astrocytes in a fibrillary background with Rosenthal fiber at the center (hematoxylin and eosin, $\times 400$ ). (C) Psammoma bodies (hematoxylin and eosin, $\times 200$ ).

prominent nucleoli. Tumor cells of similar histology were noted in the foot ulcer biopsy as well (-Fig. 4). Considering the similar features in both cerebral and foot ulcer biopsies, diagnosis of amelanotic melanoma with metastasis to the brain was made. Immunohistochemistry showed S100, Melan $\mathrm{A}$, and HMB45 positivity in tumor cells, confirming the diagnosis. The primary and metastatic tumor was surgically resected, followed by radiotherapy. The patient is symptomatically better and is on regular follow-up.

\section{Case 5}

A 59-year-old male came with a history of left hemiparesis in the last month. Biopsy was sent with a space-occupying lesion in the parietal lobe with the radiologic feature. Microscopically it showed a large area of coagulative necrosis, highly pleomorphic bizarre tumor giant cells with abundant

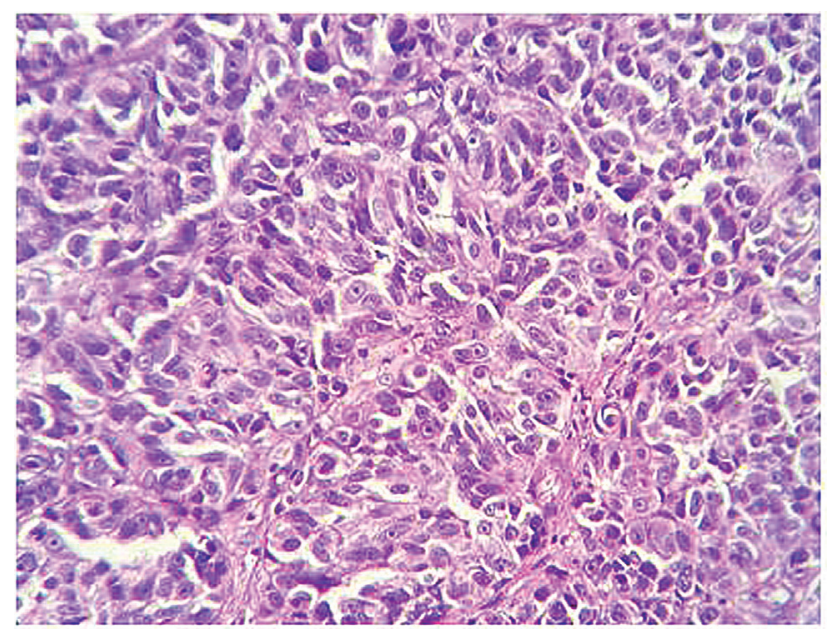

Fig. 4 Metastatic melanoma (Case 4): Sheet of tumor cell having an atypical nucleus with prominent nucleoli (hematoxylin and eosin, $\times 400$ ). 


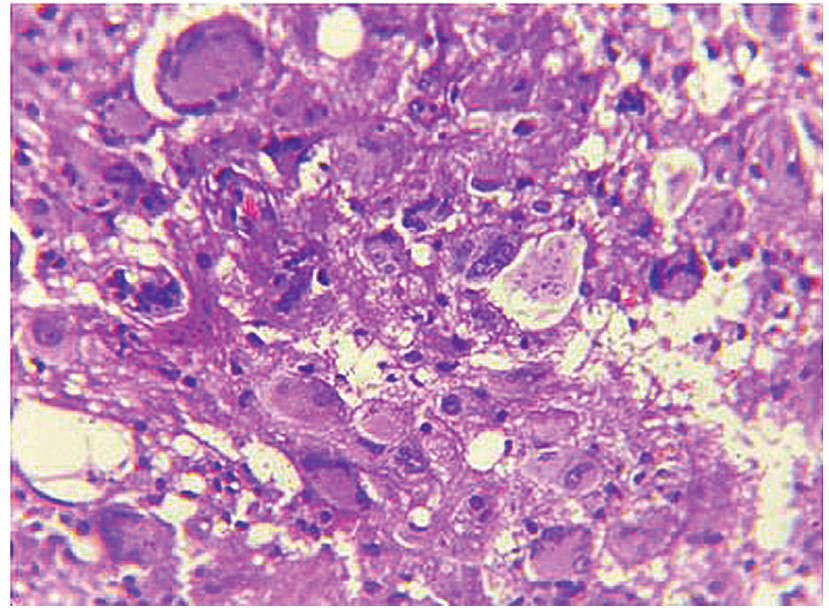

Fig. 5 Giant cell glioblastoma (Case 5): Pleomorphic tumor giant cells (hematoxylin and eosin, $\times 400$ ).

eosinophilic cytoplasm, and varying number and size of the nuclei (-Fig. 5). Histological features were that of giant cell glioblastoma (WHO grade 4). Giant cells in histology showed a diagnostic dilemma. Craniotomy was performed for evacuation of the tumor. Postoperatively patient had subarachnoid hemorrhage and pneumonia. The patient was on palliative care and later succumbed.

\section{Discussion}

The tumors of the CNS are classified according to the fifth edition of the WHO Classification of Tumors of the Central Nervous System, published in 2021..$^{5}$ Pathological diagnosis of brain tumors is very challenging. The initial evaluation with MRI is essential for diagnosis. However, even with a characteristic imaging appearance of neoplastic lesions, histologic assessment is necessary to determine the grade and genetic alternations of the tumor. ${ }^{2}$

Primary angiosarcoma is extremely rare in the CNS. Meningeal angiosarcoma and malignant meningioma may appear similar in imaging. ${ }^{6}$ Histopathologic differentials are metastasis, anaplastic meningioma, gliosarcoma, and hemangiopericytoma, differentiated from angiosarcoma using histologic primary vascular structures and immunohistochemical markers. Malignant meningiomas can show cancerous, melanomatous, or high-grade sarcomatoid patterns without expressing CD31 and FVIII factors. ${ }^{7}$ In our case (Case 1), as it was a dural-based extra-axial lesion with highly anaplastic epithelioid cells, diagnosis of anaplastic meningioma was made and sent for neuropathologists' opinion. Based on vasoformative structures on microscopy and CD31, FLI positivity, definitive diagnosis of epithelioid angiosarcoma was made. Primary angiosarcoma needs a multidisciplinary treatment, such as total tumor resection with radiotherapy or chemotherapy. The prognosis of patients with meningeal angiosarcoma remains poor. ${ }^{7}$

Based on histopathology, Case 2 was diagnosed as glioblastoma with differential diagnosis of the embryonal tumor, later diagnosed as an embryonal tumor with rhabdoid features after immunohistochemical analysis by neuropathologists. Embryo- nal tumors of the CNS are highly malignant undifferentiated or poorly differentiated tumors of neuroepithelial origin. Broad designation of CNS embryonal tumor is used for embryonal tumors without a specific diagnosis. ${ }^{8}$ Embryonal tumor differentials are anaplastic ependymoma, high-grade glioma, immature teratoma, malignant lymphoma, and Ewing sarcoma/ peripheral primitive neuroectodermal tumor (PNET), distinguished by specific cytological and architectural features and the expression of immunohistochemical markers. ${ }^{9}$ The embryonal tumor with rhabdoid cells is an atypical teratoid/rhabdoid tumor (AT/RT). Lack of expression of the SMARCB1 (INI) protein is a diagnostic tool that distinguishes RTs from other malignancies with similar histologic features. ${ }^{10}$ Some biologically aggressive tumors retain SMARCB1 protein staining. These rare tumors ( $\sim 2 \%$ of $\mathrm{AT} / \mathrm{RT}$ ) are a diagnostic challenge. Inactivation of SMARCA4 (BRG1) can be demonstrated in these cases. ${ }^{11}$ In our case, BRG1 (SMARCA4) was advised because of rhabdoid cells with retained INI1 (SMARCB1), which was not performed. Hence, the case was reported with a broad embryonal tumor designation with rhabdoid features. The current standard of care in AT/RT involves maximal safe resection, followed by intensive adjuvant chemotherapy. Postoperative radiation therapy improves overall survival (OS) but is often not advised because most patients are less than 3 years of age, which is a critical period of neurodevelopment. Outcomes among different multimodality treatment regimens vary with some long-term survivors. Despite recent improvements in therapy, the prognosis in the RT is lethal. In most studies, the median time to relapse is less than 6 months, and the median OS is less than 18 months. ${ }^{8,10}$

In Case 3, the diagnostic dilemmas were the predominant hyalinized blood vessels and extensive dystrophic calcification. MRI suggested a space-occupying lesion with hydrocephalus. The presence of sparse bipolar benign cells in a fibrillary background, absence of atypia or necrosis, occasional Rosenthal fibers at the focal area, and location of the tumor were hints for diagnosing pilocytic astrocytoma. The block was sent for neuropathologists' opinion. Although pilocytic astrocytoma is a common tumor with classical radiologic appearance, diagnostic dilemmas can arise with no classic biphasic appearance, Rosenthal fibers, and eosinophilic granular bodies, especially in patients above the second decade of life. When neoplastic cells are scant, it is difficult to distinguish these tumors from cavernous angiomas with accompanying piloid gliosis. ${ }^{12}$ Compared with other astrocytomas, totally resected pilocytic astrocytoma has an excellent prognosis. Currently accepted treatment for adult pilocytic astrocytoma is surgical gross total resection. If complete gross resection is not possible due to tumor location or medical comorbidities, subsequent radiotherapy will improve the outcome. ${ }^{13}$

Radiologically, Case 4 was high-grade glioma. The primary tumor was diagnosed simultaneously from a biopsy of a chronic nonhealing ulcer in the foot. As it was amelanotic melanoma, immunohistochemistry was advised for confirmation. Metastasis is the most common CNS tumor, and they can often be the initial manifestations of an undetected primary elsewhere. ${ }^{14}$ Metastatic tumors are often multiple and well circumscribed. However, solitary metastatic lesions mimic primary tumors in gross and radiological appearance similar to the mentioned 
case. Hence, it is crucial to consider metastasis as a differential diagnosis and to evaluate in detail for any other lesion while dealing with a case of CNS tumor, especially in high-grade tumors. Even in cases with a known distant malignancy, the possibility of an asynchronous CNS tumor should always be considered. ${ }^{3}$ Immunohistochemical analysis is most helpful for determining the exact nature and origin of the metastatic neoplasm and distinguishing primary from secondary CNS tumors. ${ }^{14}$ Surgical resection or stereotactic radiosurgery followed by systemic therapy, immunotherapy, or targeted therapy are the available treatment strategies for melanoma brain metastasis (MBM). However, MBM has a dismal prognosis with median OS of only 4 to 6 months from the diagnosis. ${ }^{15}$

Giant cell glioblastoma is characterized by a predominance of bizarre multinucleated giant cells with abundant eosinophilic cytoplasm. The definitive diagnosis is based on its histological findings and patterns. The tumor cells are positive for glial fibrillary acidic protein (GFAP), S-100, vimentin, and $\alpha-1$ antichymotrypsin. It is a WHO Grade 4 tumor, which is said to have a better survival prognosis than classical Grade 4 glioblastoma. However, established evidence in favor is lacking. ${ }^{16}$ Due to its rarity, definitive surgical management protocol for this tumor is not well known. Maximum safe resection and adjuvant radiotherapy can improve survival rate from 5 to 13 months, similar to glioblastoma multiforme patients. Early diagnosis with total resection of the tumor and adjuvant chemotherapy may increase the patient's survival. ${ }^{17}$

Diagnosis of CNS lesions is difficult with the lack of medical information. Initial pathologic diagnosis in patients with a brain tumor is mostly not the definitive diagnosis as brain neoplasms appear heterogeneous. Necrosis, vascular proliferation, and increased mitotic activity are features of high-grade tumors that may add to the diagnostic challenges. Several events lead to misdiagnosis, including errors during biopsy, incorrect interpretation of microscopic features of tumoral tissue, and significant interobserver variation during pathologic assessment. ${ }^{2}$ In this study, we have compiled five CNS tumors with a dilemma in conventional microscopic initial diagnosis where clinicoradiological correlation, immunohistochemistry, and expert opinion helped in the definitive diagnosis revealing the importance of a multidisciplinary approach while evaluating these tumors.

\section{Conclusion}

Despite significant advances in neuroimaging techniques and molecular biology, histopathologic assessment of CNS tumors remains the primary means of establishing a definitive diagnosis and predicting patient outcomes. Early diagnosis is a key for the effective management of CNS tumors. Pathologists should be familiar with radiologic features, and radiology-based provisional diagnosis should be considered during reporting. A multidisciplinary approach is essential, and pathologists should not hesitate to take expert opinion for the correct diagnosis and better treatment approach in these patients.

\section{Source(s) of Support}

Nil.

\section{Conflicting Interest}

Nil.

\section{Acknowledgment}

Authors acknowledge faculties from the Department of Neuropathology, NIMHANS, Bangalore, for their assistance in the definitive diagnosis.

\section{References}

1 Gupta T, Epari S, Moiyadi A, et al. Demographic profile, clinicopathological spectrum, and treatment outcomes of primary central nervous system tumors: retrospective audit from an academic neuro-oncology unit. Indian J Cancer 2017;54 (04):594-600

2 Anvari K, Bahadorkhan G, Samini F, Izadpanahi P, Bayatmokhtari $\mathrm{N}$, Javadinia SA. Pathological diagnostic pitfalls in the verification of brain tumors; can imaging lead to pathology alternation003F;. Rep Radiother Oncol 2015;2(04):e10598. Doi: $10.5812 /$ rro. 10598

3 Stoyanov GS, Petkova L, Dzhenkov DL. A practical approach to the differential diagnosis of intracranial tumors: gross, histology, and immunoprofile-based algorithm. Cureus 2019;11(12):e6384. Doi: $10.7759 /$ cureus.6384

4 Mohammed AA, Hamdan AN, Homoud AS. Histopathological profile of brain tumors: a 12-year retrospective study from Madinah, Saudi Arabia. Asian J Neurosurg 2019;14(04):1106-1111

5 Louis DN, Perry A, Wesseling P, et al. The 2021 WHO Classification of Tumors of the Central Nervous System: a summary. Neurooncol 2021;23(08):1231-1251

6 Malabeh Q, Abed A, Obeidat T, Abudalu R, Alwaqfi O. Primary meningeal angiosarcoma in a child: imaging findings. J $R$ Nav Med Serv 2018;25(03):66-72

7 Cai S, Wu C, Zhang S, Cai S, Wang X. Meningeal angiosarcoma: a case report and review of the literature. Transl Cancer Res 2016;5(05): 618-624

8 Shih RY, Koeller KK. Embryonal tumors of the central nervous system: from the radiologic pathology archives. Radiographics 2018;38(02):525-541

9 Cole BL, Pierson CR. Histopathologic and molecular features of central nervous system embryonal tumors for integrated diagnosis reporting. Surg Pathol Clin 2020;13(04):783-800

10 Roberts CW, Biegel JA. The role of SMARCB1/INI1 in development of rhabdoid tumor. Cancer Biol Ther 2009;8(05):412-416

11 Pfister SM, Korshunov A, Kool M, Hasselblatt M, Eberhart C, Taylor MD. Molecular diagnostics of CNS embryonal tumors. Acta Neuropathol 2010;120(05):553-566

12 Malik A, Deb P, Sharma MC, Sarkar C. Neuropathological spectrum of pilocytic astrocytoma: an Indian series of 120 cases. Pathol Oncol Res 2006;12(03):164-171

13 Lee KJ, Marchan E, Peterson J, et al. Management and survival of adult patients with pilocytic astrocytoma in the National Cancer Database. World Neurosurg 2018;112:e881-e887

14 Singh S, Amirtham U, Premalata CS, Lakshmaiah KC, Viswanath L, Kumar RV. Spectrum of metastatic neoplasms of the brain: a clinicopathological study in a tertiary care cancer centre. Neurol India 2018;66(03):733-738

15 Glitza Oliva I, Tawbi H, Davies MA. Melanoma brain metastases: current areas of investigation and future directions. Cancer J 2017;23(01):68-74

16 Shrestha S, Homagain S, Raut A, Sedhain G, Bhatta S, Shrivastav S Giant cell glioblastoma in 6-year-old kid: report of an unusual case. Clin Case Rep 2020;8(12):2936-2940

17 Belsuzarri TA, Araujo JF, Catanoce AP, et al. Giant cells glioblastoma: case report and pathological analysis from this uncommon subtype of glioma. Rare Tumors 2015;7(01):5634 